\title{
Analytical and stochastic modelling of battery cell dynamics
}

\author{
Ingemar $\mathrm{Kaj}^{1}$ and Victorien Konané2,3 \\ 1 Department of Mathematics, Uppsala University, ikaj@math.uu.se \\ 2 International Science Program, Uppsala University \\ 3 Department of Mathematics, University of Ouagadougou, Burkina Faso
}

\begin{abstract}
In this work we present and discuss a modelling framework for the basic discharge process which occurs in simple electrochemical battery cells. The main purpose is to provide a setting for analyzing delivered capacity, battery life expectancy and other measures of performance. This includes a number of deterministic and stochastic variations of kinetic battery models. The primary tool is a novel phase plane analysis of the balance of nominal and theoretical capacity. In particular, we study spatial versions of such models which lead to a linear diffusion equation with Robin type boundary conditions under scaling. Explicit solutions are obtained by considering reflected Brownian motion.
\end{abstract}

\section{Introduction}

This work concerns mathematical modeling of the state of charge and the voltage level dynamics in simple battery cells under discharge. The purpose is to provide an efficient framework for predicting battery life, delivered capacity and other measures of performance, which takes into account that batteries are commonly subject to considerable variation in performance. Such variations occur not only because of variable usage patterns or variable disload mechanisms of the electrochemically stored energy, but also as a result of recovery mechanisms in the electrolyte. The type of battery we have in mind primarily is a non-rechargeable and non-costly unit expected to last several years, such as a 3 Volts lithiumion coin battery to be deployed in large numbers for low-energy applications in communication networks, sensor networks, etc.

A battery is made of one or several electrochemical cells. The modeling discussed here relies on the simplified view that a cell essentially consists of an anode-cathode pair of electrodes connected by electrolyte, which may be liquid as in lead-acid batteries or solid as in Li-ion batteries. In the cell, stored chemical energy is converted into electrical energy through an oxidation reaction at the anode. By Faraday's first law the mass of active material altered at an electrode is directly proportional to the quantity of electrical charge which is transfered at the electrode in the battery reaction. The Nernst equation in electrochemistry then states that the logarithm of electric charge determines the terminal voltage that exists between the pair of electrode terminals. It is the terminal voltage that measures the ability of the battery to drive electric current. 
The terminal voltage for a battery in a state of rest is typically larger in magnitude than the terminal voltage under discharging due to effects of internal resistance. Batteries for digital applications would often be expected to deliver power spikes, either periodically in time or at random time points. Such pulsed discharge patterns may have a different effect on terminal voltage to continuous discharge loads of constant current.

The theoretical capacity of the battery is a measure of the maximal charge which in principle could be obtained were the battery discharged arbitrarily slowly, allowing the chemical reaction to equilibriate over time restricted only by the total amount of active material contained in the cell. The nominal capacity of a battery is typically a manufacturers specification of the amount of electric charge which is delivered if the cell is put under constant load and drained of its energy over a certain time interval. Normally the discharge process occurs on some intermediate time scale that allows for recovery mechanisms to take place. This may slow down the decrease of the state of charge or even cause the state of charge to increase. A further mechanism known to affect the performance of a battery and which we will take into account is the balance between migration and solid state diffusion. The battery stops functioning if the terminal voltage passes below a minimal acceptable level or if the battery runs out of theoretical capacity.

In addition to introducing new modeling variations our study provides a survey of a number of battery models discussed in the literature. While the simplest kinetic battery model introduced by Manwell and McGowan, [6,7], is essential for our approach as a reference and background, we also consider the spatial extension of this model, [10]. We do not discuss, however, another modeling approach based on discrete Markov chains, see e.g. [1].

We summarize the novel contributions in this work as follows. Based on an approach focusing on the interplay between remaining nominal capacity and remaining theoretical capacity during discharge evolution we perform what appears to be a novel phase plane analysis of battery capacities. This allows us to obtain battery life, gained capacity and delivered capacity as functions of the basic model parameters and in some cases to optimize performance over such parameters. The setting begins with the two-well kinetic battery ODE model of constant current discharge but includes general situations such as regular pulsed discharge or stochastic pointwise discharge. The unified approach to general workload patterns and comparison of these appears to be new. We also propose a new kinetic-diffusive battery model designed to describe the balance between migration and drift diffusion. Finally, we extend the modeling approach and generality of the models to a version where the bound charge is supposed to be distributed over a spatial reservoir.

\section{Some principles of battery cell dynamics}

To introduce the main ideas of our approach, we consider a battery which is initially fully loaded with nominal capacity $N$ and which has the theoretical 
capacity $T$ at time $t=0$. Realistically, $N \leq T$. For $t \geq 0$ let $x(t)$ denote the level of available charge and $v(t)$ the level of remaining theoretical capacity of the battery at time $t$, so $(v(0), x(0))=(T, N)$. Charge is drawn from the battery either continuously, or such that the charge level drops instantly from one discrete level to a lower level. The discharge process acts randomly or in a deterministic fashion and it acts continuously in time or at discrete time epochs.

The normalized and dimensionless quantity $\widetilde{x}(t)=x(t) / N \in[0,1]$ represents the state of charge of the battery at time $t$. A fully charged battery has $\widetilde{x}(t)=$ 1 and an empty one $\widetilde{x}(t)=0$. In practice a battery stops functioning before it runs out of charge entirely at some level $x_{0}$ which corresponds to voltage reaching a cut-off level $E_{c}$. According to the Nernst equation the concentration $C$ of active material at an electrode determines the potential $E$ according to $E=E_{0}-K_{e} \ln C$, where $E_{0}$ is the equilibrium potential and $K_{e}=R T_{a} / n F$ with $R$ the ideal gas constant, $T_{a}$ absolute temperature, $n$ the valency of the battery reaction ( $n=1$ for Lithium), and $F$ Faraday's constant. Faraday's law identifies the capacity $Q$ of the cell as a multiple of $C$, with a proportionality constant which is $F$ times volume. Since $\widetilde{x}(t)$ is a measure of capacity we obtain the terminal voltage $E_{t}$ of the cell at time $t$, as

$$
E_{t}=E_{0}+K_{e} \ln \widetilde{x}(t), \quad 0 \leq t \leq t_{0},
$$

where $t_{0}$ is the battery life given by the first instance at which $E_{t}$ reaches the cut-off voltage $E_{c}<E_{0}$. For example, a Li-ion battery may have $E_{0}=3$ and $E_{c}=2$ Volt.

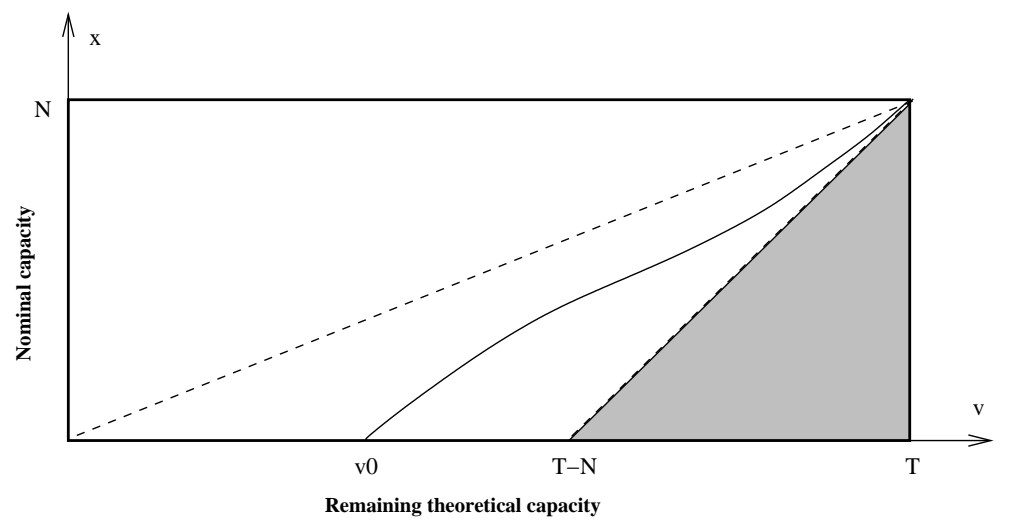

Fig. 1. Phase-plane trace of nominal and theoretical capacities

It is natural to consider the trajectory of the system $(v(t), x(t)), t \geq 0$, as a path in the $(v, x)$ phase plane starting in $(T, N)$ at $t=0$. Initially, the path moves downwards and to the left in the $(v, x)$ plane as the nominal and hence the remaining theoretical capacity decreases. While the remaining theoretical 
capacity continues its descent with the same average rate as the discharge process, it is reasonable that the battery recovers some nominal charge capacity. This is because chemical transport in the electrolytes enables previously stored material to become available, at least if $\lambda$ is not too large. This effect is likely to be less effective at lower levels of nominal charge.

If there exists a solution $v_{0}>0$ of $x(v)=x_{0}$ then $v_{0}$ is the remaining capacity at the battery charge expiration time. For this case we note that $D=T-v_{0}$ is the delivered capacity of the battery. We expect, based on the brief discussion above, that $D$ tends to $T$ if $\lambda \rightarrow 0$. Also, we normalize the nominal capacity such that $D$ tends to $N$ if $\lambda \rightarrow \infty$, c.f. [9], Figure 6 . A closely related quantity is the gain of the battery, $G=T-N-v_{0}$. This is the capacity which is gained during the life of the battery and measures the amount of bound charge that the battery was able to convert into available charge and deplete during its time of operation. Figure 1 indicates a typical trace in the phase-plane starting from $(T, N)$ and ending in $\left(v_{0}, 0\right)$.

\section{The Kinetic battery model}

The Kinetic Battery Model, [10], is a deterministic modeling approach which assumes that charge is drawn continuously over time according to a given discharge current $i(t) \geq 0, t \geq 0$. The average discharge rate is $\bar{\lambda}=\lim _{t \rightarrow \infty} \frac{1}{t} \int_{0}^{t} i(s) d s$, if this limit exists. The most basic case is a battery subject to constant discharge over time, $i(t)=\lambda$. The total theoretical capacity of the battery is split in two components called available charge and bound charge. As above, for $t \geq 0$ let $v(t)$ denote the total capacity and $x(t)$ the available capacity of the battery. Call $y(t)=v(t)-x(t)$ the bound charge. Suppose $x(0)=N, v(0)=T>N$.

The kinetic battery model involves a parameter $c \in(0,1)$ which allows the ratios $x(t) / c$ and $y(t) /(1-c)$ to be interpreted as the current heights of an available charge well and a bound charge well, respectively. During operation of the battery, bound charge is supposed to be made available according to a rate which is proportional to the height difference $y(t) /(1-c)-x(t) / c$ between the bound and available charge wells. The matching with initial conditions then dictates one should take $c=N / T \in(0,1)$ to be the fraction of total theoretical capacity which is initially made available. Then at time $t=0$, the wells have equal height $T$ and the charge flow gradient which builds up between the two wells represents recovery of the battery, in the sense of its positive growth effect on the nominal charge level $x$. As a result we obtain for $(x(t), y(t))$ the linear system of differential equations

$$
\begin{cases}x^{\prime}(t)=-i(t)+k\left(\frac{y(t)}{1-c}-\frac{x(t)}{c}\right), & x(0)=N \\ y^{\prime}(t)=-k\left(\frac{y(t)}{1-c}-\frac{x(t)}{c}\right), & y(0)=T-N,\end{cases}
$$


where $k$ is a conductance parameter. Hence the total discharge process is independent of the charge flow gradient and we have

$$
v(t)=x(t)+y(t)=T-\int_{0}^{t} i(s) d s, \quad t \geq 0 .
$$

The linear system is readily solved in terms of the parameters $k, T$ and $c=N / T$, as

$$
\left\{\begin{array}{l}
x(t)=c v(t)-(1-c) \int_{0}^{t} e^{-k(t-s) / c(1-c)} i(s) d s \\
y(t)=(1-c) v(t)+\int_{0}^{t} e^{-k(t-s) / c(1-c)} i(s) d s .
\end{array}\right.
$$

Since $c v(t)-x(t) \geq 0$ the system life equals

$$
t_{0}=\inf \left\{t>0: x(t)=x_{0} \text { or } v(t)=0\right\}=\inf \left\{t>0: x(t)=x_{0}\right\}
$$

The model in this form is discussed in e.g. [5].

Phase plane analysis and general workload discharge. It is straightforward to extend the kinetic battery model and incorporate general discharge patterns by replacing $i(t) d t$ with some measure $\Lambda(d t)$, and consider the differential system

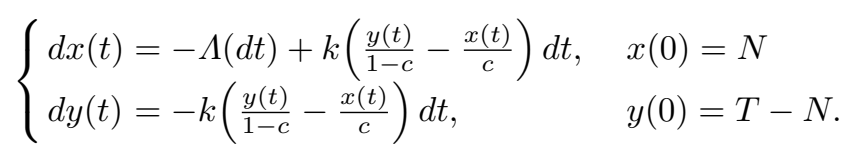

Then $v(t)=x(t)+y(t)=T-\Lambda(t)$ and

$$
\left\{\begin{array}{l}
x(t)=c v(t)-(1-c) \int_{0}^{t} e^{-k(t-s) / c(1-c)} \Lambda(d s) \\
y(t)=(1-c) v(t)+\int_{0}^{t} e^{-k(t-s) / c(1-c)} \Lambda(d s) .
\end{array}\right.
$$

For example, if a cell is subject to successive periods of low, medium and high loads then $\Lambda(d t)$ would be a continuous measure with discharge rates varying accordingly from one time interval to the next.

Next we analyze in some detail and compare three types of discharge patterns for the kinetic battery model in its extended form. First of all, for the constant discharge case $\Lambda(t)=\lambda t$ we obtain the solution $\left(v_{\lambda}(t), x_{\lambda}(t)\right)$, where $v_{\lambda}(t)=$ $T-\lambda t$ and

$$
x_{\lambda}(t)=c v_{\lambda}(t)-\lambda c(1-c)^{2} k^{-1}\left(1-e^{-k t / c(1-c)}\right) .
$$

In line with the phase plane view point in Figure 1, the corresponding trajectory of the bivariate dynamical system $\left(v_{\lambda}(t), x_{\lambda}(t)\right)$ in the $(v, x)$-plane with terminal condition $x(T)=N$ is

$$
x(v)=c v-C_{\lambda}\left(1-e^{-k(T-v) / \lambda c(1-c)}\right), \quad v \leq T, \quad C_{\lambda}=\lambda c(1-c)^{2} / k .
$$

This system starts in $(v, x)=(T, N)$ at time $t=0$ and traces out a path below the diagonal $x=c v$ but above the line $x=-(T-N)+v$ which exits at time $t_{0}$ in 
$\left(v_{0}, x_{0}\right)$, where $0 \leq v_{0} \leq T-N$. The phase plane path depends on the parameters $\lambda$ and $k$ only through the ratio $\lambda / k$. Figure 2 shows the drop of voltage according to $E_{t}=3+0.2 \ln (x(t) / N)$ as a function of normalized capacity $1-v(t) / T$ (upper panel) and phase plane curves (lower panel) until the cut-off voltage of 2 Volts is reached, which occurs close to the time of complete discharge where $x(t) \approx 0$. Three discharge rates, $\lambda / k=500,1000,2500$ are indicated and compared to the ideal case $x=c v$ for a very small discharge current, which corresponds to $100 \%$ utilization, and the worst case of highest loads where $40 \%$ of the available capacity is utilized.
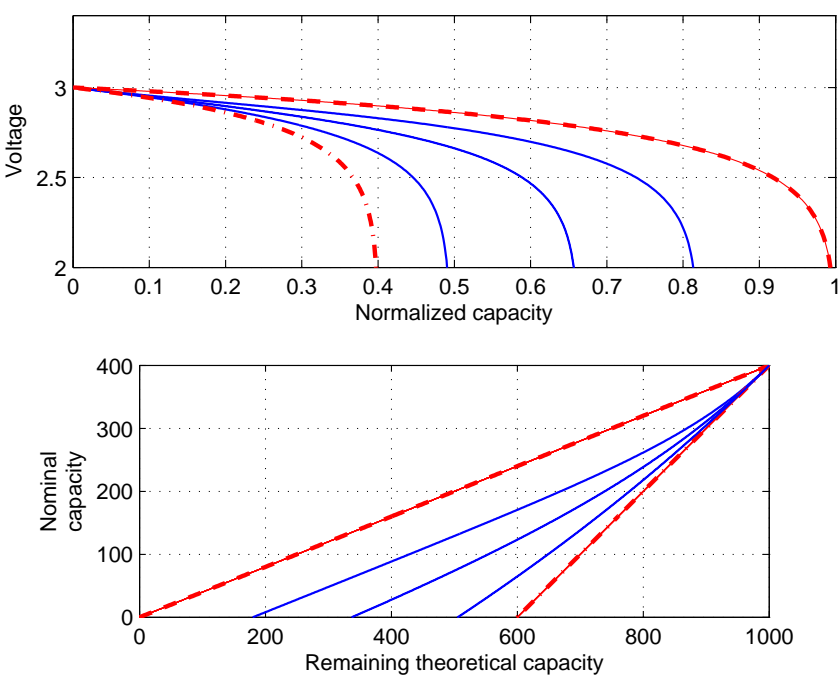

Fig. 2. Upper panel: Voltage versus (normalized) capacity; Lower panel: Discharge profiles for the kinetic battery model; Parameters: constant current load, $N=400$, $T=1000, \lambda / k=500,1000,2500$

Another case of interest is regularly spaced pulsed discharge. This is a relevant model for batteries in sensor nodes programmed to carry out a fixed task once per day, say. Here we let $r>0$ be the time between any two consecutive pulses each releasing the charge $\lambda r$. The corresponding discharge measure is $\Lambda(t)=$ $\lambda r \sum_{j=1}^{\lfloor t / r\rfloor} \delta_{j r}$, for which the average discharge rate is kept at (approximately) $\lambda t$. The solution $\left(x^{(r)}(t), v^{(r)}(t)\right)$ for this case is

$$
\begin{aligned}
& v^{(r)}(t)=T-\Lambda(t) \approx T-\lambda t \\
& x^{(r)}(t)=c v^{(r)}(t)-(1-c) \lambda r \sum_{j=1}^{\lfloor t / r\rfloor} e^{-k(t-j r) / c(1-c),}
\end{aligned}
$$


where evaluation of the geometric sum $\sum_{j=1}^{\lfloor t / r\rfloor}\left(e^{k r / c(1-c)}\right)^{j}$ yields

$$
x^{(r)} \approx c v^{(r)}-C_{\lambda}^{(r)}\left(1-e^{-k\left(T-v^{(r)}\right) / \lambda c(1-c)}\right), \quad C_{\lambda}^{(r)}=\frac{\lambda r(1-c)}{1-e^{-k r / c(1-c)}} .
$$

The previous case $\Lambda(t)=\lambda t$ is recovered by taking an informal limit $r \rightarrow 0$.
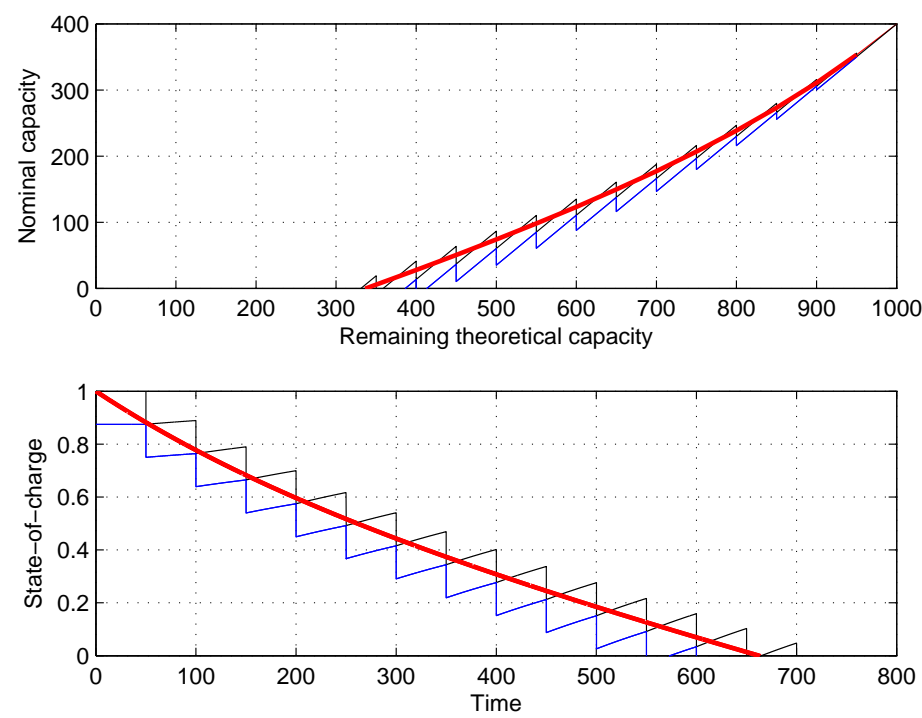

Fig. 3. Discharge of pulsed kinetic battery model, $r=50, N=400, T=1000, \lambda / k=$ 1000

Strictly speaking, the curves with constants $C_{\lambda}^{(r)}$ indicate the lower jump points of the discharge profile. This is appropriate as the battery is considered empty at the first instance when the voltage drops below $E_{c}$. Figure 3 shows phase plane and the change of state with time for the kinetic battery model under pulsed discharge with $r=50$ and additional parameters $N=400, T=1000$, $\bar{\lambda} / k=1000$. Two curves are shown for the case when the first pulse occurs at $t=0$ (blue) or $t=r$ (black) and compared with the constant discharge case of same average (red).

As a third example we let $(\Lambda(t))_{t \geq 0}$ be a Poisson process with intensity measure $\lambda d t$. This is a battery released of charge pulsewise at random times uniformly scattered over the time interval of operation with an average of $\lambda$ per time unit. The result is a system of random processes $(V(t), X(t))$ with $V(t)=T-\Lambda(t)$ and

$$
X(t)=c V(t)-(1-c) Z(t), \quad Z(t)=\int_{0}^{t} e^{-k(t-s) / c(1-c)} d \Lambda(s),
$$


and $Z(t)$ is known as a so called Poisson shot-noise process. Clearly, the expected value $E X(t)=x_{\lambda}(t)$ is given by (1). Moreover, the shot-noise process has a steady-state $Z_{\infty}$, such that asymptotically

$$
Z(t) \Rightarrow Z_{\infty}, \quad E Z_{\infty}=\lambda c(1-c) / k, \quad \operatorname{Var} Z_{\infty}=\lambda c(1-c) / 2 k .
$$

Kinetic-diffusive battery model The kinetic battery model was primarily framed for lead-acid batteries. Shortcomings of the model have been discussed in e.g. [3], and attempts have been made to incorporate other designs. In the context of Ni-MH batteries, [9] proposed a modified, non-linear, factor in the flow charge between the two wells.

For Li-ion cells a shortcoming of the kinetic battery model appears to be that solid state diffusion is not taken into account. In solid phase the application of an external driving force makes the diffusing particles experience a drift motion in addition to random diffusion. This effect of diffusion drift of charge carriers is discussed in detail in the specialized electrochemical litterature on all-solid batteries and is known to hamper performance of the units, see e.g. [2]. We propose the following modification of the dynamics of the two-well kinetic battery model as a means of introducing in a simplistic but meaningful way a negative drift in the flux of charge:

$$
\left\{\begin{array}{l}
d x(t)=-\Lambda(d t)+k\left((1-p)\left(\frac{y(t)}{1-c}-\frac{x(t)}{c}\right)-p \frac{1}{c}\left(\frac{N}{c}-\frac{y(t)}{1-c}\right)\right) d t, \quad x(0)=N \\
d y(t)=-k\left((1-p)\left(\frac{y(t)}{1-c}-\frac{x(t)}{c}\right)-p \frac{1}{c}\left(\frac{N}{c}-\frac{y(t)}{1-c}\right)\right) d t, \quad y(0)=T-N
\end{array}\right.
$$

Here, $p, 0 \leq p \leq 1$, signifies a fraction of the current flow of charge which is removed and sent back to the bound well. The solution in this case is given by

$$
\left\{\begin{aligned}
x(t) & =(c+(1-c) p) v(t)-(1-c) p T-(1-p)(1-c) \int_{0}^{t} e^{-k(t-s) / c(1-c)} \Lambda(d s) \\
& =(1-p)\left(c v(t)-(1-c) \int_{0}^{t} e^{-k(t-s) / c(1-c)} \Lambda(d s)\right)+p(v(t)-(T-N)) \\
v(t) & =x(t)+y(t)=T-\Lambda(t)
\end{aligned}\right.
$$

Now choose a discharge measure $\Lambda(d t)$. It is then straightforward to derive results such as $(2,3)$ for the more general model that involves the drift parameter $p$.

Performance of the kinetic battery model Here we compare briefly delivered capacity and battery life for the kinetic battery model. For simplicity we consider the standard model $p=0$. All formulas listed in this section may also be derived for the case $0<p<1$ of the kinetic-diffusive modification discussed above. Indeed, we conclude this section with some comments on delivered capacity for the general model.

We begin with the non-random models. The unused capacity that remains after depletion of all available charge is the unique solution $v_{0}>0$ of $x(v)=x_{0}$. The delivered capacity is $D=T-v_{0}$ and the gained capacity $G=D-N$. By (3) and (2), which we include as the case $r=0$,

$$
x_{0}=c v_{0}-C_{\lambda}^{(r)}\left(1-e^{-k\left(T-v_{0}\right) / \lambda c(1-c)}\right), \quad C_{\lambda}^{(0)}=C_{\lambda} .
$$


Equation (5) may be solved explicitly in terms of the so called Lambert W function, the principal branch of which we denote by $W_{0}$. Then

$$
v_{0}=\frac{x_{0}+C_{\lambda}^{(r)}}{c}-\frac{\lambda c(1-c)}{k} W_{0}\left(\frac{k C_{\lambda}^{(r)}}{\lambda c^{2}(1-c)} \exp \left\{-\frac{k\left(T-x_{0} / c-C_{\lambda}^{(r)}\right)}{\lambda c(1-c)}\right\}\right)
$$

However, for typical parameters the exponential term in (5) may be ignored for $v$ close to $v_{0}$, and hence $v_{0} \approx\left(x_{0}+C_{\lambda}^{(r)}\right) / c$. In conclusion, the delivered capacity $D_{\lambda}^{(r)}$ for the deterministic version of the kinetic battery model is approximately

$$
D_{\lambda}^{(r)} \approx T-x_{0} / c-C_{\lambda}^{(r)} / c
$$

The lifelength $t_{0}$ of the battery is directly proportional to the delivered capacity. Indeed, since $\left(v\left(t_{0}\right), x\left(t_{0}\right)\right)=\left(v_{0}, x_{0}\right)$ and $v(t)=T-\lambda t$ we have $\lambda t_{0}=D$. As an example, the lifelength for the continuous model (2) is obtained as the solution $t_{0} \in[T / \lambda-1 / k, T / \lambda]$ of

$$
t_{0}=\frac{T}{\lambda}-\frac{x_{0}}{c \lambda}-\frac{1}{k}(1-c)^{2}\left(1-e^{-k t_{0} / c(1-c)}\right) .
$$

Again this equation may be solved in terms of $W_{0}$, as

$$
t_{0}=\frac{T}{\lambda}-\frac{x_{0}}{c \lambda}-\frac{1-c}{k}+\frac{c(1-c)}{k} W_{0}\left(\frac{1}{c} \exp \left\{-\frac{k\left(T-x_{0} / c\right)}{\lambda c(1-c)}+\frac{1}{c}\right\}\right) .
$$

Turning to the random model driven by Poisson discharge events, if we stop at the random time $t_{0}=\min \left\{t: X(t)=x_{0}\right\}$, then by $(4)$,

$$
c V\left(t_{0}\right) \approx x_{0}+(1-c) Z_{\infty} \approx x_{0}+(1-c) E Z_{\infty}=x_{0}+C_{\lambda}
$$

Hence

$$
\Lambda\left(t_{0}\right) \approx T-\frac{x_{0}+C_{\lambda}}{c}=T\left(1-\frac{x_{0}+C_{\lambda}}{N}\right)
$$

and so the distribution of the lifelength $t_{0}$ is comparable to a Gamma distribution $\Gamma(m, \lambda)$ where $m$ is an integer approximation of $T\left(1-\left(x_{0}+C_{\lambda}\right) / N\right)$. Also, the average delivered capacity for the Poisson model is given by $D_{\lambda}=T\left(1-\left(x_{0}+\right.\right.$ $\left.\left.C_{\lambda}\right) / N\right)$.

As mentioned above it is straightforward to include the kinetic-diffusive version. The delivered capacity will decrease with increasing $p$ as illustrated in Figure 4 , with $p=0, p=0.2$ and $p=0.4$. For the basic model $p=0$, the delivered capacity $D_{\lambda}$ descends from its maximal value $T$, or $T\left(1-x_{0} / N\right)$ in case $x_{0}>0$, to the asymptotic value $N$ as $\lambda \rightarrow \infty$. For $p>0$ however there is a maximal $T_{p} \approx\left(N-x_{0}\right) /((1-p) c+p)$ which is attained for vanishing load, $\lambda \rightarrow 0$. The interpretation is that solid phase diffusion puts a principal restriction on the amount of chemical energy which can be drawn from the cell. 


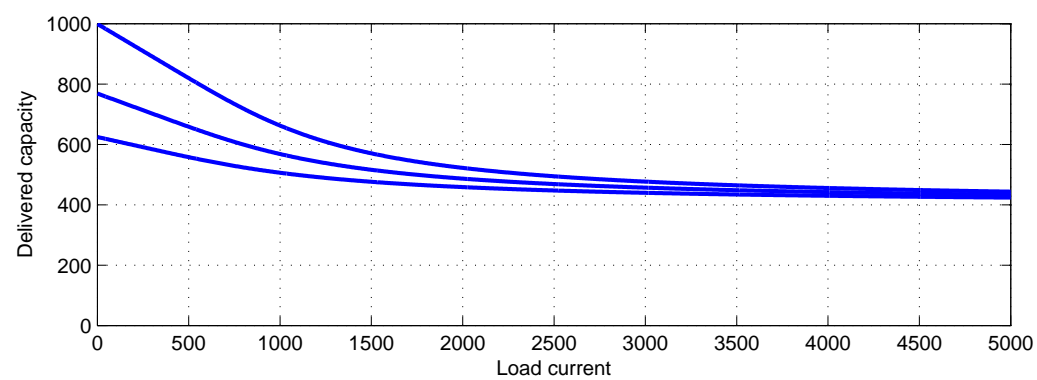

Fig. 4. Delivered capacity $D$ as function of current load $\lambda / k$ for $p=0$ (upper), $p=0.2$ (mid) and $p=0.4$ (lower); $T=1000, N=400$,

\section{Spatial diffusion models}

This approach extends the mechanism of the kinetic battery model to act pairwise on adjacent fluid compartments spread out on a one-dimensional spatial range. We begin with a discretized version and split the charges in $m$ components $u(t)=\left(u_{1}(t), \ldots, u_{m}(t)\right)$. Here $u_{1}$ is the available charge, $u_{2}$ is a bound well charge for $u_{1}$ and so on until $u_{m}$, which is a bound well charge for $u_{m-1}$. As charge is drawn from $u_{1}$ electrochemical material continuously flows downwards from each bound well to help recover the charge level at the next lower well. By considering a limit of many small wells we obtain a limiting PDE for the charge concentration profile, which may be solved explicitly and analyzed by phase plane methods just as for the two-well case.

Spatial version of the kinetic battery model By treating each pair of adjacent components as available and bound wells, we obtain the coupled system of linear equations

$$
\left\{\begin{array}{c}
d u_{1}(t)=-\Lambda(d t)+k_{c}\left(c u_{2}(t)-(1-c) u_{1}(t)\right) d t \\
d u_{2}(t)=-k_{c}\left(c u_{2}(t)-(1-c) u_{1}(t)\right) d t+k_{c}\left(c u_{3}(t)-(1-c) u_{2}(t)\right) d t \\
\quad \vdots \\
\begin{array}{c}
d u_{m-1}(t)=-k_{c}\left(c u_{m-1}(t)-(1-c) u_{m-2}(t)\right) d t \\
\quad+k_{c}\left(c u_{m}(t)-(1-c) u_{m-1}(t)\right) d t
\end{array} \\
d u_{m}(t)=-k_{c}\left(c u_{m}(t)-(1-c) u_{m-1}(t)\right) d t
\end{array}\right.
$$

where we have put $k_{c}=k / c(1-c)$ as a temporary notation. Rewriting,

$$
\left\{\begin{array}{c}
d u_{1}(t)=-\Lambda(d t)+\frac{k_{c}}{2}\left(u_{2}(t)-u_{1}(t)\right) d t+k_{c}(c-1 / 2)\left(u_{1}(t)+u_{2}(t)\right) d t \\
d u_{2}(t)=\frac{k_{c}}{2}\left(u_{1}(t)-2 u_{2}(t)+u_{3}(t)\right) d t+k_{c}(c-1 / 2)\left(u_{3}(t)-u_{1}(t)\right) d t \\
\quad \vdots \\
\begin{array}{c}
d u_{m-1}(t)=\frac{k_{c}}{2}\left(u_{m-2}(t)-2 u_{m-1}(t)+u_{m}(t)\right) d t \\
\quad+k_{c}(c-1 / 2)\left(u_{m}(t)-u_{m-2}(t)\right) d t
\end{array} \\
d u_{m}(t)=-\frac{k_{c}}{2}\left(u_{m}(t)-u_{m-1}(t)\right)-k_{c}(c-1 / 2)\left(u_{m-1}(t)+u_{m}(t)\right) d t
\end{array}\right.
$$


To prepare for studying the limit as $m \rightarrow \infty$ we introduce a new parameter $\ell$, that will be tuned to the initial condition $u(0)$ and $N$ and $T$ later, and think of each well as occupying intervals of length $\ell / m$ positioned uniformly on the strip $0 \leq x \leq \ell$. To this end, put $\varepsilon=\ell / m$, and for $x=j \varepsilon, j=1, \ldots, m$, let $u_{\varepsilon}(t, x)=u_{j}(t)$. We also adapt conductivity by introducing the scaled parameter $\kappa=k / m^{2}$. Moreover, put $\kappa_{c}=\kappa / c(1-c)$. Then, for $x \in\{2 / m, \ldots,(\ell-1) / m\}$,

$$
\begin{aligned}
d u_{\varepsilon}(t, x)= & \frac{\kappa_{c} \ell^{2}}{2} \frac{u_{\varepsilon}(t, x-\varepsilon)-2 u_{\varepsilon}(t, x)+u_{\varepsilon}(t, x+\varepsilon)}{\varepsilon^{2}} d t \\
& +\kappa_{c} \ell m(2 c-1) \frac{u(t, x+\varepsilon)-u(t, x-\varepsilon)}{2 \varepsilon} d t .
\end{aligned}
$$

The boundary equations attain the form

$$
\begin{aligned}
\frac{d u_{\varepsilon}(t, \varepsilon)}{m}= & -\frac{\Lambda(d t)}{m} \\
& +\frac{\kappa_{c}}{2}\left\{\ell \frac{u_{\varepsilon}(t, 2 \varepsilon)-u_{\varepsilon}(t, \varepsilon)}{\varepsilon}+m(2 c-1)\left(u_{\varepsilon}(t, \varepsilon)+u_{\varepsilon}(t, 2 \varepsilon)\right)\right\} d t
\end{aligned}
$$

and

$$
\frac{d u_{\varepsilon}(t, \ell)}{m}=-\frac{\kappa_{c}}{2}\left\{\ell \frac{u_{\varepsilon}(t, \ell)-u_{\varepsilon}(t, \ell-\varepsilon)}{\varepsilon}+m(2 c-1)\left(u_{\varepsilon}(t, \ell-\varepsilon)+u_{\varepsilon}(t, \ell)\right)\right\} d t
$$

We now consider the case of scaling the height parameter $c=c_{m}$ with the number of spatial compartments by putting $c_{m}=(1+\mu / m) / 2$. With $\mu \neq 0$ and large $m$, this will keep the system close to the symmetric situation $c_{m} \sim 1 / 2$ but with a flux of charge at each adjacent pair of wells with magnitude of order $\mu / m$. This gives the approximative system

$$
\begin{aligned}
d u_{\varepsilon}(t, x)= & -\Lambda(d t) \delta_{\varepsilon}(d x)+2 \kappa \ell^{2} \frac{u_{\varepsilon}(t, x-\varepsilon)-2 x_{\varepsilon}(t, x)+u_{\varepsilon}(t, x+\varepsilon)}{\varepsilon^{2}} d t \\
& +4 \kappa \ell \mu \frac{u(t, x+\varepsilon)-u(t, x-\varepsilon)}{2 \varepsilon} d t
\end{aligned}
$$

with Robin type boundary conditions

$$
\ell \frac{u_{\varepsilon}(t, 2 \varepsilon)-u_{\varepsilon}(t, \varepsilon)}{\varepsilon}=-2 \mu u_{\varepsilon}(t, \varepsilon), \quad \ell \frac{u_{\varepsilon}(t, \ell-\varepsilon)-u_{\varepsilon}(t, \ell)}{\varepsilon}=2 \mu u_{\varepsilon}(t, \ell-\varepsilon) .
$$

Taking an informal limit as $m \rightarrow \infty$, we conclude that the relevant limiting equation is

$$
\begin{aligned}
& d u(t, x)=-\Lambda(d t) \delta_{0}(d x)+2 \kappa \ell^{2} \frac{\partial^{2} u}{\partial x \partial x}(t, x) d t+4 \kappa \ell \mu \frac{\partial u}{\partial x}(t, x) d t, \quad 0 \leq x \leq \ell \\
& \ell \frac{\partial u}{\partial x}(t, 0+)=-2 \mu u(t, 0), \quad \ell \frac{\partial u}{\partial x}(t, \ell-)=2 \mu u(t, \ell), \quad u(0, x)=u_{0}(x) .
\end{aligned}
$$

Our interpretation is that $u(t, 0)_{t \geq 0}$ represents the available charge of the battery and $\{u(t, x), 0<x<\ell\}_{t \geq 0}$ represents the fluid level of a reservoir of bound 
charge such that $\int_{(0, \ell)} u(t, x) d x$ is what remains in the reservoir at time $t$. For simplicity we will consider the case $u_{0}(y)=u_{0}, y \in[0, \ell]$, for which initial charge is uniformly located on the strip $[0, \ell]$. Hence

$$
v(t)=u(t, 0)+\int_{0}^{\ell} u(t, x) d x, \quad t \geq 0, \quad v(0)=u_{0}+u_{0} \ell
$$

defines the remaining capacity in the system as function of time.

Now we are in position to relate the model parameters $u_{0}$ and $\ell$ to the battery parameters $N$ and $T$. For this we take the initial level of available charge to be $u_{0}=N$ and the initial content of the reservoir to be $u_{0} \ell=T-N$. Then the total potentially available charge is $v(0)=T$ and we have $\ell=T / N-1$.

Consider the parameters $\sigma^{2}=4 \kappa \ell^{2}$ and $\beta=-4 \kappa \ell \mu$. Let $\left(\xi_{t}\right)_{t \geq 0}$ denote a Brownian motion with variance parameter $\sigma^{2}$ and constant drift $\beta$. The initial condition is $\xi_{0}=x \in(0, \ell)$ and the paths are subject to reflecting boundaries at both end points 0 and $\ell$ with no loss of probability mass. Denote by $p_{\ell}(t, y, x)$ the corresponding transition probability density function, such that $P\left(\xi_{t} \in d x \mid \xi_{0}=y\right)=p_{\ell}(t, y, x) d x$. Then the solution $u(t, x)$ of the above PDE has the representation

$$
u(t, x)=\int_{0}^{\ell} u_{0}(y) p_{\ell}(t, y, x) d y-\int_{0}^{t} p_{\ell}(t-s, 0, x) \Lambda(d s) .
$$

The reflected Brownian motion $\left(\xi_{t}\right)$ arises also as a model in economics and other fields, see e.g. [11] and references in there. The transition density is given by

$$
\begin{aligned}
& p_{\ell}(t, y, x)=\frac{2 \mu}{\ell} \frac{e^{-2 \mu x / \ell}}{1-e^{-2 \mu}}+\frac{2 e^{-\mu(x-y) / \ell}}{\ell} \times \\
& \sum_{n=1}^{\infty}\left(\cos \left(\frac{n \pi x}{\ell}\right)-\frac{\mu}{n \pi} \sin \left(\frac{n \pi x}{\ell}\right)\right)\left(\cos \left(\frac{n \pi y}{\ell}\right)-\frac{\mu}{n \pi} \sin \left(\frac{n \pi y}{\ell}\right)\right) \frac{e^{-2 \kappa\left(\mu^{2}+n^{2} \pi^{2}\right) t}}{1+(\mu / n \pi)^{2}} .
\end{aligned}
$$

In particular, for the symmetric case, taking $\mu \rightarrow 0$,

$$
p_{\ell}(t, y, x)=\frac{1}{\ell}+\frac{2}{\ell} \sum_{n=1}^{\infty} \cos (n \pi x / \ell) \cos (n \pi y / \ell) e^{-2 \kappa n^{2} \pi^{2} t} \text {. }
$$

By (6),

$$
\begin{aligned}
& u(t, x)=(T-N-\lambda t) \frac{2 \mu}{\ell} \frac{e^{-2 \mu x / \ell}}{1-e^{-2 \mu}} \\
& +4 N \mu e^{-\mu x / \ell} \sum_{n=1}^{\infty}\left(\cos \left(\frac{n \pi x}{\ell}\right)-\frac{\mu}{n \pi} \sin \left(\frac{n \pi x}{\ell}\right)\right) \frac{(-1)^{n} e^{\mu}-1}{\mu^{2}+n^{2} \pi^{2}} \frac{e^{-2 \kappa\left(\mu^{2}+n^{2} \pi^{2}\right) t}}{1+(\mu / n \pi)^{2}} \\
& -\frac{\lambda e^{-\mu x / \ell}}{\kappa \ell} \sum_{n=1}^{\infty}\left(\cos \left(\frac{n \pi x}{\ell}\right)-\frac{\mu}{n \pi} \sin \left(\frac{n \pi x}{\ell}\right)\right) \frac{n^{2} \pi^{2}\left(1-e^{-2 \kappa\left(\mu^{2}+n^{2} \pi^{2}\right) t}\right)}{\left(\mu^{2}+n^{2} \pi^{2}\right)^{2}}
\end{aligned}
$$


The remaining capacity is

$$
v(t)=u(t, 0)+\int_{0}^{\ell} u(t, x) d x=u(t, 0)+T-N-\lambda t .
$$

Furthermore,

$$
\begin{aligned}
u(t, 0)= & (T-N-\lambda t) \frac{1}{\ell} \frac{2 \mu}{1-e^{-2 \mu}}-\frac{\lambda}{\kappa \ell} \sum_{n=1}^{\infty} \frac{n^{2} \pi^{2}\left(1-e^{-2 \kappa\left(\mu^{2}+n^{2} \pi^{2}\right) t}\right)}{\left(\mu^{2}+n^{2} \pi^{2}\right)^{2}} \\
& +4 N \mu \sum_{n=1}^{\infty}\left((-1)^{n} e^{\mu}-1\right) \frac{n^{2} \pi^{2} e^{-2 \kappa\left(\mu^{2}+n^{2} \pi^{2}\right) t}}{\left(\mu^{2}+n^{2} \pi^{2}\right)^{2}} .
\end{aligned}
$$

This shows that the quantities $(v, u)=(v(t), u(t, 0))$ form an autonomous system such that the relation between $v$ and $u=u(v)$ is given by

$$
\begin{aligned}
u= & (v-u) \frac{1}{\ell} \frac{2 \mu}{1-e^{-2 \mu}}-\frac{\lambda}{\kappa \ell} \sum_{n=1}^{\infty} \frac{n^{2} \pi^{2}\left(1-e^{-2 \kappa\left(\mu^{2}+n^{2} \pi^{2}\right)(T-N-v+u) / \lambda}\right)}{\left(\mu^{2}+n^{2} \pi^{2}\right)^{2}} \\
& +4 N \mu \sum_{n=1}^{\infty}\left((-1)^{n} e^{\mu}-1\right) \frac{n^{2} \pi^{2} e^{-2 \kappa\left(\mu^{2}+n^{2} \pi^{2}\right)(T-N-v+u) / \lambda}}{\left(\mu^{2}+n^{2} \pi^{2}\right)^{2}} .
\end{aligned}
$$

As $\mu \rightarrow 0$,

$$
u=\frac{1}{\ell}(v-u)-\frac{\lambda}{\kappa \ell} \sum_{n=1}^{\infty} \frac{1}{n^{2} \pi^{2}}\left(1-e^{-2 \kappa n^{2} \pi^{2}(T-N-v+u) / \lambda}\right) .
$$

The important conclusion now is that we have obtained closed phase plane representations of nominal and theoretical capacity also for the spatial model, at least for constant load. Thus, performance can be studied just as for the two-well model. Figure 5 displays typical discharge profiles of the driftless spatial version of the kinetic battery model. The graphs are very similar to those for the basic model in Figure 2. Figure 6 shows the effect of adding drift $\mu$ to the model. With the same $N$ and $T$ as previously and for $\lambda / \kappa=2000$, three discharge profile curves are plotted with $\mu=-0.5, \mu=0$ and $\mu=0.5$. Clearly, negative drift lowers the delivered capacity whereas positive drift $\mu>0$ improves the utilization of bound charge.

Spatial version of the kinetic-diffusive battery model Finally we consider a spatial extension of the proposed kinetic-diffusive model with symmetric kinetic dynamics $c=1 / 2$ but diffusive effect governed by $p \geq 0$. Here, the total conductivity has been partitioned into two mechanisms, see [8]. First the basic flow of charge caused by the height difference between wells, secondly a drift under the action of the discharge load which affects the bound charge. The parameter $p$ controls the balance of these two contributions to the overall flux. 


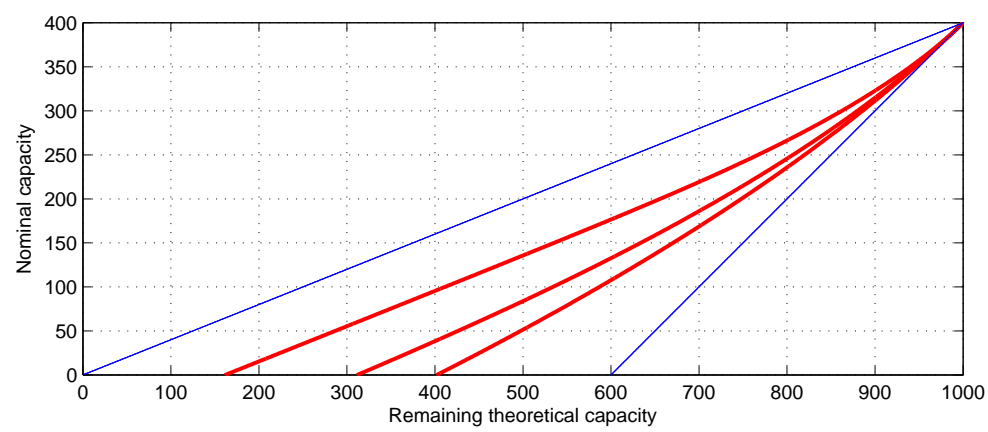

Fig. 5. Discharge profiles of the spatial kinetic battery model, $N=400, T=1000$, from left to right: $\lambda / \kappa=1000,2000,3000$

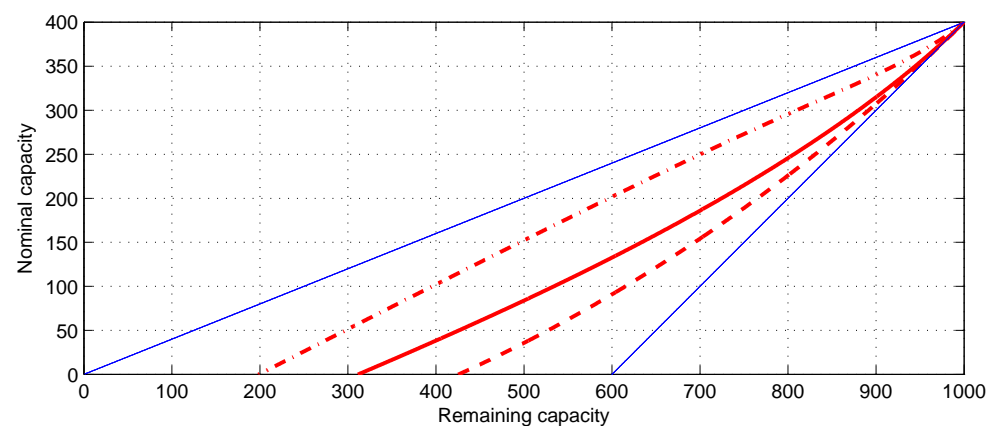

Fig. 6. Discharge profiles of the spatial kinetic battery model with drift, $N=400$, $T=1000, \kappa=1, \lambda=2000, \mu=-0.5,0,0.5$

Putting $q=1-p$,

$$
\left\{\begin{array}{l}
d u_{1}(t)=-\Lambda(d t)+2 k q\left(u_{2}(t)-u_{1}(t)\right) d t-4 k p\left(N-u_{2}(t)\right) d t \\
d u_{2}(t)=2 k q\left(u_{1}(t)-2 u_{2}(t)+u_{3}(t)\right) d t+4 k p\left(u_{3}(t)-u_{2}(t)\right) d t \\
\quad \vdots \\
d u_{m-1}(t)=2 k q\left(u_{m-2}(t)-2 u_{m-1}(t)+u_{m}(t)\right) d t+4 k p\left(u_{m}(t)-u_{m-1}(t)\right) d t \\
d u_{m}(t)=-2 k q\left(u_{m}(t)-u_{m-1}(t)\right)+4 k p\left(N-u_{m}(t)\right)
\end{array}\right.
$$

Again, we place $m$ wells of width $\ell / m$ on the interval $0 \leq x \leq \ell$. With $\varepsilon=\ell / m$ and $u_{\varepsilon}(t, x)=u_{j}(t)$ for $x=j \varepsilon, j=1, \ldots, m$, and with scaled parameters $\kappa=k / m^{2}>0$ and $\rho=m p>0$, we find for for $x \in\{2 / m, \ldots,(\ell-1) / m\}$,

$$
\begin{aligned}
d u_{\varepsilon}(t, x)= & 2 \kappa \ell^{2}(1-\rho / m) \frac{u_{\varepsilon}(t, x-\varepsilon)-2 u_{\varepsilon}(t, x)+u_{\varepsilon}(t, x+\varepsilon)}{\varepsilon^{2}} d t \\
& +4 \kappa \ell \rho \frac{u(t, x+\varepsilon)-u(t, x)}{\varepsilon} d t .
\end{aligned}
$$


The boundary equations attain the form

$\frac{d u_{\varepsilon}(t, \varepsilon)}{m}=-\frac{\Lambda(d t)}{m}+2 \kappa\left\{\ell(1-\rho / m) \frac{u_{\varepsilon}(t, 2 \varepsilon)-u_{\varepsilon}(t, \varepsilon)}{\varepsilon}-2 \rho\left(N-u_{\varepsilon}(t, 2 \varepsilon)\right)\right\} d t$

and

$$
\frac{d u_{\varepsilon}(t, \ell)}{m}=-2 \kappa\left\{\ell(1-\rho / m) \frac{u_{\varepsilon}(t, \ell)-u_{\varepsilon}(t, \ell-\varepsilon)}{\varepsilon}-2 \rho\left(N-u_{\varepsilon}(t, \ell-\varepsilon)\right)\right\} d t .
$$

Considering a limit for large $m$, this yields

$$
\begin{aligned}
& d u(t, x)=-\Lambda(d t) \delta_{0}(d x)+2 \kappa \ell^{2} \frac{\partial^{2} u}{\partial x \partial x}(t, x) d t+4 \kappa \ell \rho \frac{\partial u}{\partial x}(t, x) d t, \quad 0 \leq x \leq \ell \\
& \ell \frac{\partial u}{\partial x}(t, 0+)=2 \rho(N-u(t, 0)), \quad \ell \frac{\partial u}{\partial x}(t, \ell-)=-2 \rho(N-u(t, \ell)),
\end{aligned}
$$

with initial condition $u(0, x)=u_{0}(x)$. In comparison to the previous case where a drift $\mu$ was created by keeping the height parameter $c$ asymptotically of the order $c=1 / 2+\mu / 2 m$, in this case we obtain the same equation with $\rho$ replacing $\mu$ but other boundary conditions still of the generalized Robin type.

\section{References}

1. C.F. Chiasserini, R.R. Rao: Energy efficient battery management. IEEE J. Sel. Areas in Communication 19:7, 2001.

2. Danilov, D., Niessen, R.A.H., Notten, P.H.L.: Modeling All-Solid-State Li-Ion Batteries. Journal of the Electrochemical Society, 158:3, A215-A222 (2011)

3. Jongerden, M.R., Haverkort, B.R., Bohnenkamp, H.C. and Katoen, J.P.: Maximizing System Lifetime by Battery Scheduling. In: Proceedings of the 39th Annual IEEE/IFIP International Conference on Dependable Systems and Networks, IEEE Computer Society Press, (2009)

4. Jongerden, M.R., Haverkort, B.R.: Which battery model to use? IEEE/IET Software, 3:6, 445-457 (2009)

5. Jongerden, M.R. and Haverkort, B.R., Lifetime improvement by battery scheduling. In: 27th Annual UK Performance Engineering Workshop, UKPEW (2011)

6. Manwell, J., McGowen, J.: Lead acid battery storage model for hybrid energy systems. Solar Energy 50, 399-405 (1993).

7. J. Manwell, J. McGowen: Extension of the kinetic battery model for wind/hybrid power systems. Proc. fifth European Wind Energy Association Conf. 284-289 (1994).

8. Mehrer, H.: Diffusion in Solids: Fundamentals, Methods, Materials, DiffusionControlled Processes. Springer-Verlag, Berlin Heidelberg (2007)

9. V Rao, G Singhal, A Kumar, N Navet: Battery model for embedded systems. 18th International Conference on VLSI Design (2005)

10. Rakhmatov, D., Vrudhula, S.: Energy Management for Battery-Powered Embedded Systems. ACM Transactions on Embedded Computing Systems, 2:3, 277324 (2003)

11. Veestraeten, D.: The conditional probability density function for a reflected Brownian motion. Computational Economics 24, 185-207 (2004) 